\title{
Characteristic Analysis of Adjustable Permanent Magnetic Coupler with Slotted Conductor Disk
}

\author{
Qingzhong Gao ${ }^{1,2}$, Dazhi Wang ${ }^{1}$ and Sheng Lin ${ }^{1,2}$ \\ 1 College of Information Science and Engineering, Northeastern University, \\ China \\ 2 Academy of Automation, Shenyang Institute of Engineering, China \\ * Corresponding author: gaoqingzhong_xia@163.com
}

\begin{abstract}
It is essential to optimize the conductor surface vortex path because the efficiency of Permanent magnet governor depends on the excitation level of the induced current. In order to further improve efficiency, this article focuses on a permanent magnet governor with slotted conductor disk. The $2 D$ and $3 D$ dynamic simulation model are established and the characteristics of slotted and plain structure are analyzed. The results are shown that the vortex path of the permanent magnet governor with slotted structure is optimized, the magnetic flux leakage is reduced, and the thermal capacity is higher. The transmission torque mathematical model with temperature parameters is established considering the transmission torque. The transmission torque is affected by conductivity changing with the conductor disk heating. The effects of temperature changing and relative speed on torque transmission are analyzed. Finally, the torque characteristics of slotted and plain structure were compared by the experimental test under different conditions. It is proved that the slotted structure can improve the transmission efficiency and reduce the vortex field impact due to the temperature of conductor disk rising. And it is verified that the mathematical model is valid.
\end{abstract}

Keywords: adjustable permanent magnetic coupler, slotted conductor, finite element, stray current, moving eddy current field

\section{Introduction}

The adjustable permanent magnetic coupler has been the hot topic in the researches conducted by domestic and foreign scholars since 1999. [1] Currently it has been applied to industries such as pumps and blowers. [2] In comparison with frequency convertors with efficiency at $95 \%$, its efficiency is a little bit lower (93\%) but it has remarkable advantages: it uses purely mechanical non-contact type of structure to effectively remove power harmonic pollution, electromagnetic interference, avoid transmission between motor and load through vibration and truly realize green and energy conservation. In order to further improve the efficiency of the adjustable permanent magnetic coupler, it is necessary to deal with the stray current on conductor disc and optimize eddy current path. The above-mentioned currents form eddy current consumption through the heat produced on the conductor disc and the generated heat not only wastes energy but also disturbs the magnetic performance of permanent magnet and reduces the system's ability in torque transmission.

In recent years, scholars have conducted researches on permanent magnet transmission with certain achievements. In Document [3], it conducts analysis and comparison of magnetic circuit arrangement in plain structure and it points out to select relevant parameters to effectively control the amount of eddy current loss. In Document [4], it sets up the 3D finite element model of eddy current field for plain structure, calculates 
electromagnetic field distribution and summarizes the principle of selecting major parameters. In Document [5], it sets up 3D moving eddy current field's mathematical model for plain permanent eddy current coupler, analyzes the distribution of magnetic flux in conductor and distribution of eddy current and finishes the optimized design for relevant parameters of magnetic circuit. The above-mentioned researches focus on optimizing parameters to improve transmission performance through analyzing model and simulation result without structural improvement based on practice. Secondly, both analysis model and simulation model are simplified with consideration of only the influence on transmission performance by major parameters and it fails to consider the influence brought by factors such as temperature, vibration and mal-alignment, etc.

In this paper, it puts forward a new type of conductor disc design method. In this design, ladder-shaped slot exists in conductor disc for the convenience of promoting the flowing of eddy current along the appropriate path, optimize eddy current path and avoid the production of stray current. Besides, it also helps improve the adjustable permanent magnetic coupler improve its radiation ability and reduce the influence on performance brought by temperature. In this study, firstly, it is necessary to use ANSYS Finite Element Analysis Software to set up the 2D dynamic simulation model for adjustable permanent magnetic coupler with plain/slotted structure. It will conduct the simulation results of the magnetic field distribution in these two structures to verify the high efficiency of slotted structure. Secondly, it is necessary to set up mathematical model and 3D simulation model for the slotted structure to analyze distribution principle of 3D moving eddy current field, magnetic field and thermal energy and exhibit the optimized result of eddy current path. Finally, it will use the experiment to verify the effectiveness of improving torque transmission in slotted structure and the precision of the calculation method.

\section{Working Principle and Mechanical Design}

The structure of adjustable permanent magnetic coupler with slotted structure is as illustrated in Diagram 1 (a) and it is mainly divided into two parts: the first part is the conductor disc connected with motor; the second part is the permanent magnetic disc connected with the load terminal. Use copper disc as the conductor disc. Permanent disc is composed of aluminum disc, steel disc and $\mathrm{NdFeB}$ permanent Magnet and there is air gap between it and the doctor disc. When motor is rotating, conductor disc will cut magnetic curves to form eddy current circuit in the surface so as to generate conduction magnetic field. It is necessary to the mutual functions of magnetic fields to enable the permanent magnetic rotator keep rotating with load and conductor disc in the same direction. Load end is connected with air gap adjustment device. Besides, adjust two permanent rotators to conduct anti-clockwise action so as to change the gap between airs and to keep output rotating speed under control.

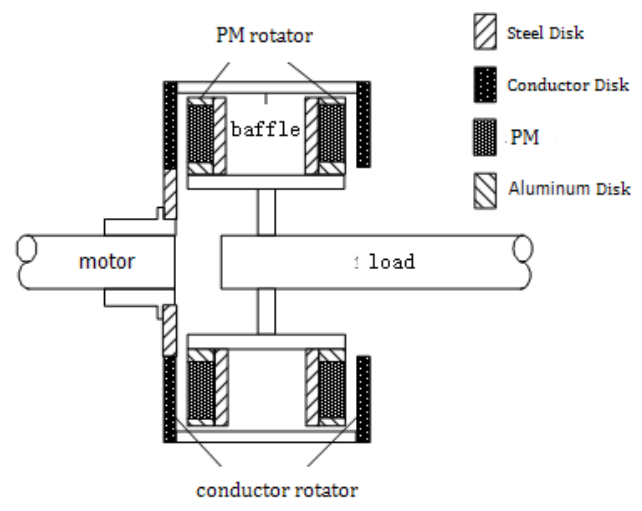

(a) Sectional View of Adjustable Permanent Magnetic Coupler 


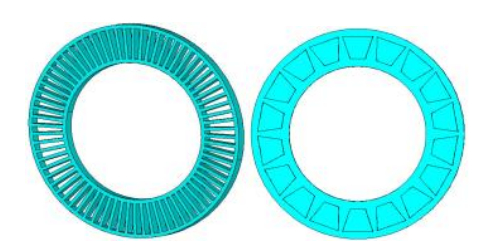

(b) Slotted Conductor Disc and Permanent Magnetic Disc

\section{Figure 1. Structure Diagram of Adjustable Permanent Magnetic Coupler}

In order to optimize vortex circulation path, it opens 72 ladder-shaped slots on the conductor disc as illustrated in Figure 1 (b). In the Figure, the ladder-shaped slot dimension is as follows: upper edge $-8 \mathrm{~mm}$; lower edge $-4 \mathrm{~mm}$; height $-40 \mathrm{~mm}$; thickness - $10 \mathrm{~mm}$. In order to cooperate with the vortex circuit on copper disc, the permanent magnet uses ladder-shaped magnet with $\mathrm{N}$ and $\mathrm{S}$ poles alternatively arrayed on the steel backing disc. In Figure 1 (b), the dimension of the ladder-shaped magnet is as follows: upper edge $-40 \mathrm{~mm}$; lower edge $-20 \mathrm{~mm}$; height $-30 \mathrm{~mm}$; thickness $-20 \mathrm{~mm}$. select the same surface, use ANSYS finite element analysis software to establish 2D dynamic simulation model for adjustable permanent magnetic coupler with slotted structure and conduct analysis of amount of the relevant electromagnetic field. Simulation parameters are as follows: steel disc length $-80.28 \mathrm{~mm}$; thickness $-10 \mathrm{~mm}$; copper disc length -$80.28 \mathrm{~mm}$; thickness $-10 \mathrm{~mm}$; air gap $-3 \mathrm{~mm}$; motor output rotation speed -- $1455 \mathrm{r} / \mathrm{min}$. Figure 2 (a) describes how magnetic line passes slotted copper disc and finishes the closed magnetic circuit. In comparison with Figure 2 (b), slotted structure effectively improves the flux density and reduces magnetic leakage in magnetic circuit.

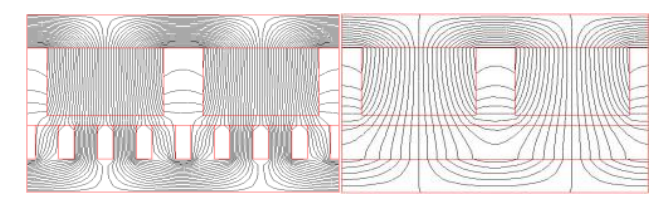
(a) Slotted Structure
(b) Plain Structure

\section{Figure 2. 2D Magnetic Field Map of Two Structures}

\section{Analytical Model of Transmission Torque in Slotted Structure}

In the process of calculating $\mathrm{T}$ - magnetic transmission torque, in the angle of calculus, we can treat the copper disc as the combination of infinite copper stripes with multiple lengths (r2-r1) sharing the same center of circle in tight arrangement. When the adjustable permanent magnetic coupler is working, the existence of slip makes the relative angular speed of copper disc at $\omega$ and the intensification of magnetic induction vertically going through the copper disc is at B. Eddy current is induced on copper disc. Relative to permanent magnetic plate rotation, copper is equal to cutting numerous magnetic forces of lines conducted by loaded conduction lines to produce magnetic transmission torque on the copper disc as illustrated in Figure 3:

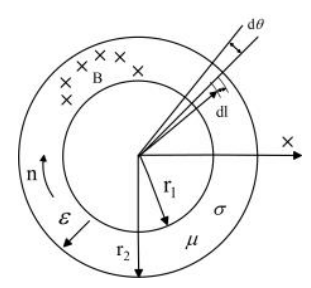

Figure 3. Map of Copper Disc 
In which, $\mathrm{dl}$ is copper disc's radial infinitesimal element; $\mathrm{d} \theta$ is the copper disc's circumferential infinitesimal element; $\varepsilon$ is motional electromotive force on copper disc; Based on Document [6], we can see that effective value Ie of eddy current on copper disc is:

$$
I_{e}=\frac{\sigma B v}{2}
$$

In which, $v$ is the relative linear speed of copper disc and permanent magnetic plate with $v=\omega n l$ and unit at $\mathrm{m} / \mathrm{s}$. $\omega n$ is the relative angular speed of copper disc and permanent magnetic plate. $\sigma$ is conductivity. Eddy current di within $d \theta$ curve on copper disc is:

$$
d i=\frac{I_{e}}{2 \pi} \phi
$$

In which, $\mathrm{d} \theta$ is the copper disc's circumferential infinitesimal element with unit at rad;

Based on Ampere's law, we can obtain Ampere's force dF and magnetic transmission torque $\mathrm{dT}$ at length of $\mathrm{dl}$ on the copper disc is:

$$
\begin{aligned}
& d F=B d i d l \\
& d T=l d F
\end{aligned}
$$

In which, $\mathrm{dl}$ is copper disc's radial infinitesimal element with unit at $\mathrm{m} ; \mathrm{l}$ is radial torque of the infinitesimal element with unit at m; introduce formula (2), (3) and (4) in it and we can obtain:

$$
d T=\frac{B I_{e}}{2 \pi} l d \theta d l
$$

Based on integration of (5), we can obtain magnetic transmission torque Tat:

$$
T=\int_{r_{1}}^{r_{2}} \int_{0}^{2 \pi} \frac{B I_{e}}{2 \pi} l d \theta d l
$$

Introduce (1) to (6) and simplify it to obtain:

$$
T=\frac{\sigma B^{2} \omega_{n}\left(r_{2}^{3}-r_{1}^{3}\right)}{6}
$$

The eddy current produced on the copper disc radiates heat to lower conductivity $\sigma$ of copper disc. When temperature change is large, the calculation of transmission torque needs to consider the influence brought by changes of temperature on $\sigma$. The formula for $\sigma$ with the change of temperature is as follows:

$$
\sigma=\frac{1}{\rho_{0}(1+\alpha t)}
$$

In which, $\rho_{0}$ is resistivity at zero degree with value at $1.678 \times 10-8$ and $\alpha$ is temperature coefficient with value at $0.004 ; \mathrm{t}$ is centigrade temperature. Introduce formula (8) to (7) and we can obtain transmission torque in different temperatures as follows:

$$
T=\frac{B^{2} \omega_{n}\left(r_{2}^{3}-r_{1}^{3}\right)}{6 \rho_{0}(1+\alpha t)}
$$




\section{Slotted Structure's Finite Element and Characteristic Analysis}

\subsection{Modeling}

Magnetic field generated by eddy current changes with the time [7-9] and it belongs to time-varying field. In low frequency, effect of displacement current is omitted and we can obtain basic equation as follows:

$$
\left\{\begin{array}{l}
\nabla \times \boldsymbol{H}=\boldsymbol{J} \\
\nabla \times \boldsymbol{E}=-\frac{\partial \boldsymbol{B}}{\partial \boldsymbol{t}} \\
\boldsymbol{\nabla} \cdot \boldsymbol{B}=0
\end{array}\right.
$$

In the formula: $\mathrm{H}-$ magnetic field intensity, $\mathrm{A} / \mathrm{m} ; \mathrm{J}-$ conduction current density, $\mathrm{A} / \mathrm{m} 2 ; \mathrm{B}$ - magnetic flux density, T.

Additional constituent equation:

$$
\left\{\begin{array}{l}
\boldsymbol{J}=\sigma \boldsymbol{E} \\
\boldsymbol{B}=\mu \boldsymbol{H}
\end{array}\right.
$$

In the formula, $\sigma$-conductivity; $\mathrm{E}$ - electric field strength, $\mathrm{V} / \mathrm{m} ; \mu$-magnetic permeability. Flux density B can be displayed by magnetic vector potential A and the expression is as follows:

$$
\boldsymbol{B}=\boldsymbol{\nabla} \times \boldsymbol{A}
$$

There is no source current input in the adjustable permanent magnetic coupler, there is no source current density in overall domain and there is only vertex density on surface of copper ring. Vortex density expression is as follows:

Adopt A, $\Phi$-A Method [10-11] to establish the mathematical model of vortex field and in overall domain, it is divided into vortex area $\mathrm{V} 1$, permanent magnet area $\mathrm{V} 2$ and air area V3. The control equation in each area is as follows:

$$
\begin{gathered}
\mathrm{V} 1: \nabla \times\left(\frac{1}{\mu} \nabla \times \boldsymbol{A}\right)+\sigma \nabla \phi-\sigma[\boldsymbol{V} \times(\nabla \times \boldsymbol{A})]=0 \\
\mathrm{~V} 2: \nabla \times \frac{1}{\mu}\left(\nabla \times \boldsymbol{A}-\boldsymbol{B}_{r}\right)=0 \\
\mathrm{~V} 3: \nabla \times \frac{1}{\mu}(\nabla \times \boldsymbol{A})=0
\end{gathered}
$$

In the formula: $\mathrm{Br}$-residual flux density of permanent magnet; based on the above-mentioned control equation group, we can see that the moving speed of conduct $\mathrm{V}$ is a constant and there is no derivative t related to time. Impose flux on the peripheral boundary of overall domain the parallel boundary condition and use magnetic vector potential A and scalar potential $\Phi$ to find the flux density B, current density $\mathrm{J}$ and other amount of magnetic field in the area.

In this paper, it uses ANSYS software to conduct 3D dynamic finite element modeling for adjustable permanent magnetic coupler with slotted structure and it analyzes the relevant magnetic field amount. [12-15] the permanent magnet selects $\mathrm{NdFeB}$ with specification of $\mathrm{N} 38 \mathrm{H}$; copper disc uses brass with model number at $\mathrm{H} 62$ and steel disc uses No.45 steel. As to structure dimension, in addition to using 2D simulation parameters, the other relevant parameters are as follows: copper disc internal radius $-90 \mathrm{~mm}$, external 
radium $140 \mathrm{~mm}$; steel disc internal radius $-90 \mathrm{~mm}$, external radium $140 \mathrm{~mm}$; amount of permanent magnet -18 ; number of ladder-shaped slots -72 ; motor output rotation speed -- 1455r/min; 3D dynamic simulation model for slotted structure and plain structure is as illustrated in Figure 4:
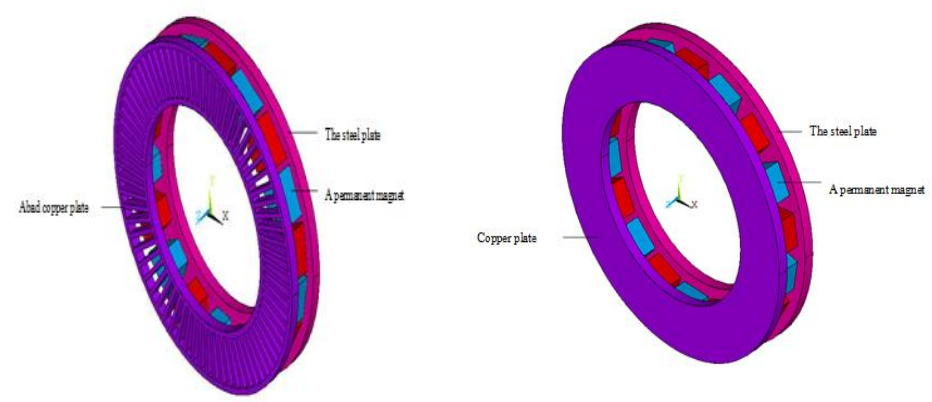

Figure 4. Simulation Model for Slotted Structure

\subsection{Characteristic Analysis}

Figure 5 (a) describes eddy current distribution of slotted copper disc. [16] Based on the figure we can know that: eddy currents are mainly distributed on the thin layer of the copper disc surface (facing magnet side) and in skin depth, they gradually descend along the axial direction; the eddy currents are not evenly distributed on copper disc surface and they mainly concentrate on radial bar especially in places adjacent to internal radius and external radius where there are obvious skin effect; the circuit of eddy current is ladder shaped; the amount of circuits corresponds with amount of poles; the current directions in two adjacent circuits are opposite; in comparison with plains structure, eddy current path has obvious optimized effect with remarkably reduced stray current; current flows along the radial direction and it helps enhance dragging force and effectively improve eddy current density.

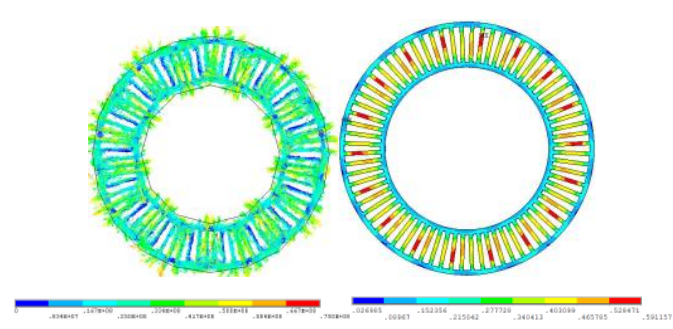

(a) Eddy Current Distribution (b) Magnetic Field Distribution

\section{Figure 5. Distribution of Eddy Current on Slotted Copper Disk}

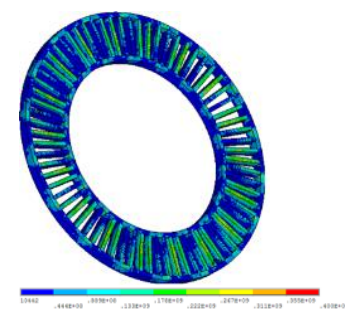

(a) Heat Distribution

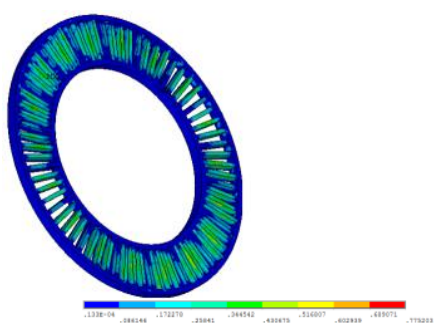

(b) Magnetic Field's Braking Force Distribution

Figure 6. Heat of Magnetic Field Braking Force Distribution in Slotted Copper Disk 
Figure 5 (b) describes the induction magnetic field distribution on the slotted copper disc. Based on the figure we can know that: induction magnetic fields mainly exist on copper disc surface and in skin depth and they gradually descend along the axial direction; induction magnetic field on copper disc surface is not evenly distributed and it mainly concentrates on radial bar in ladder shape with amount corresponding with amount of eddy current circuits; magnetic fields exhibit periodic sinusoidal distribution in circumferential direction and the magnetic field at the magnetic core is the most powerful one;

Figure 6 (a) describes the heat distribution on the slotted copper disc. Based on the figure we can know that: heat distribution corresponds with eddy current circuit and it mainly concentrates on radial bar on surface of the slotted copper disc especially on the crossing path of the adjacent eddy current circuits. In comparison with heat distribution in plain copper disc, the heat on surface of the slotted copper disc is lower. The slot design helps the heat radiation of adjustable permanent magnetic coupler.

Figure 6 (b) describes distribution of the magnetic field braking force imposed on the slotted copper disc. Based on the figure we can know that: magnetic field braking force imposed on the slotted copper disc mainly concentrates on radial bar on surface of the slotted copper disc; the magnetic field force is at its maximum number at average radius and it is at its minimum number at internal/external radius; in comparison with heat distribution in plain copper disc, the slot design on the copper disc deals with stray current in eddy current circuit, optimizes eddy current path and enhances flux density in magnetic path, by which it effectively elevates the magnetic field braking force imposed on the slotted copper disc for the benefit of producing larger transmission torque.

\section{Experimental Test}

The general structure of the experimental bench is as illustrated as that in Figure 7. The main equipment includes base board, inverter, AC motor, permanent magnet governor prototype, torque/speed meter, DC motor and DC speed regulator, among which base board is used for support and fixation; AC motor is used as prime mover with rated power at $15 \mathrm{kw}$ and rated rotation speed at $1455 \mathrm{r} / \mathrm{min}$; DC motor and DC speed regulator are used for simulating load with rated power at $15 \mathrm{kw}$; torque/speed meter are used to measure the torque and rotation speed output from adjustable permanent magnetic coupler.

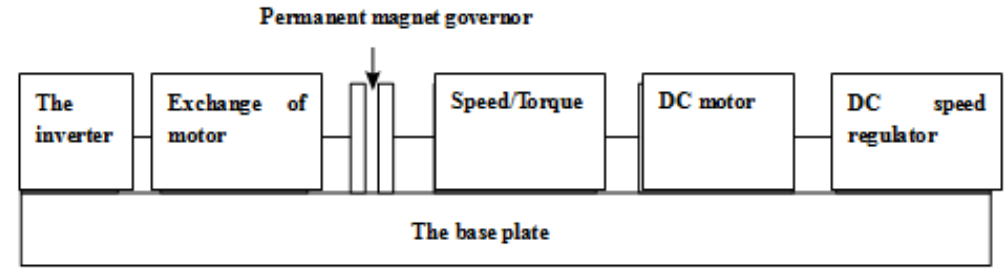

\section{Figure 7. Structure of Adjustable Permanent Magnetic Coupler Test Bench}

Experimental text result is as illustrated as that in Figure 8. It describes the influence on torque brought by length of air gap in slotted structure. Based on the comparison of the experimental data of two structures, we can see that under the same air gap, the conductor disc with slotted structure can effectively improve transmission torque at $10 \%$. 


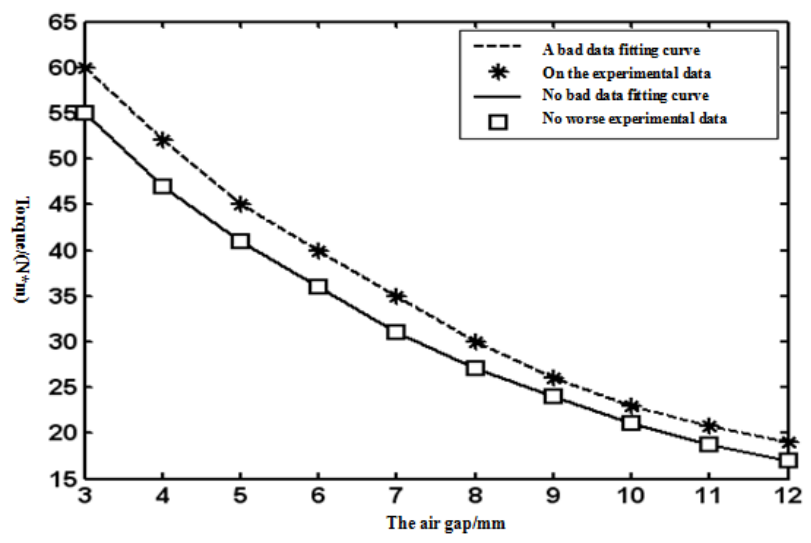

Figure 8. Air Gap-Torque Characteristic Curve for Slotted Structure

Figure 9 describes the influence on transmission torque brought by the number of ladder-shaped slots on the slotted copper disc. Based on the figure, we can see that with the increase of the number of ladder-shaped slots, the transmission torque keeps increasing; if the number of ladder-shaped slots is too small, the slotted conductor disc cannot play the role of increasing transmission torque. Instead, it will reduce transmission torque. Only when the number of ladder-shaped slots is above 60, the excellence of slotted circuit structure can be expressed. In this condition, the transmission torque in this structure is obviously elevated in comparison with regular plain circuit structure.

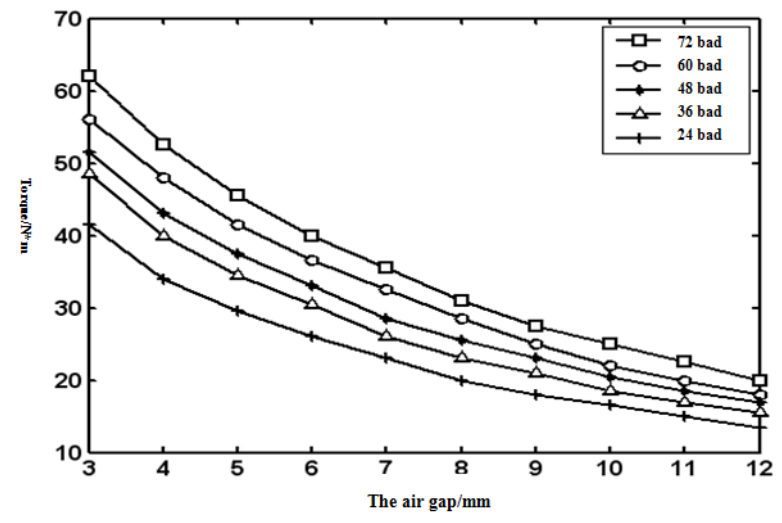

Figure 9. Effect of Slot Number on Torque

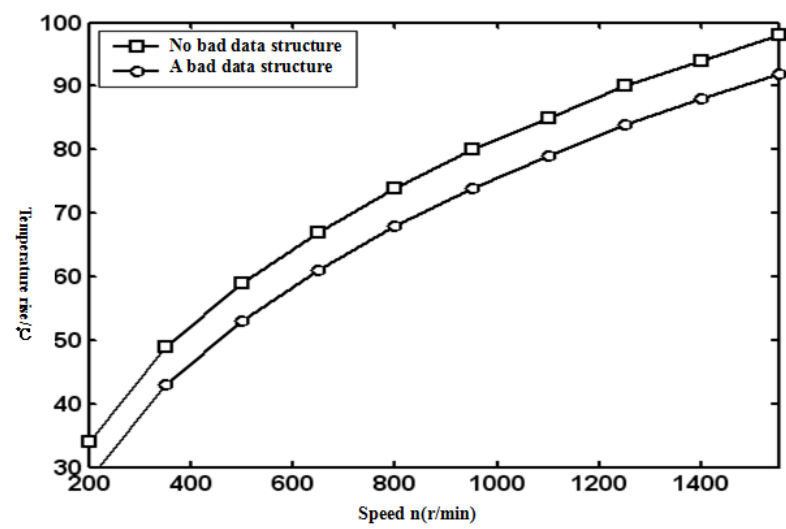

Figure 10. Experimental Data Fitting of Speed and Temperature Rise 
Use infrared radiation thermometer to record the temperature rise values of copper disc in different rotation speeds. With the increase of rotation speed, the eddy current produced by cutting magnetic lines is also enlarged and it leads to the continuous temperature rise in working environment. Based on Figure 10, we can see that the ladder-shaped slots in slotted structure enhance the heat radiation ability. In comparison with plain structure, they are more beneficial for reducing the temperature in working environment and they just reduce the hazards of demagnetizing the permanent magnet due to the high temperature.

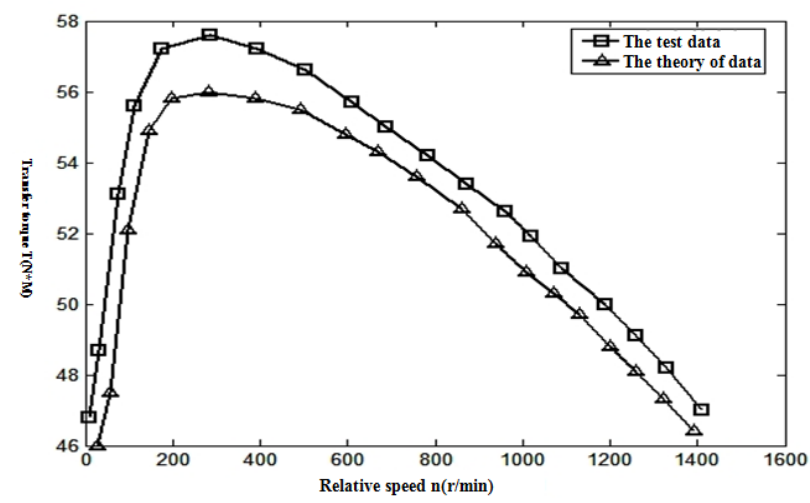

Figure 11. The Curve of Relative Speed and Transmission Torque

We can obtain temperature data in different rotation speeds based on Figure 10. Introduce the data in Formula 9 and we can calculate the theoretical data of relative speed and transmission torque. As what is illustrated in Figure 11, in low speed, transmission torque increases with the increase of rotation speed; after the speed reaches the critical value, with the elevation of rotation speed, transmission torque gradually descends because of the demagnetizing effect generated on vortex of the copper disc. Based on Figure 11, we can see that the calculated value is little bit smaller than the experimental value due to the ignorance of influence brought by hysteresis loss, radial vortex density and vortex density in cutting direction. There is difference between theoretical value and experimental value but the difference lies in the acceptable range and the difference mainly comes from the corresponding simplification and assumption in theoretical analysis.

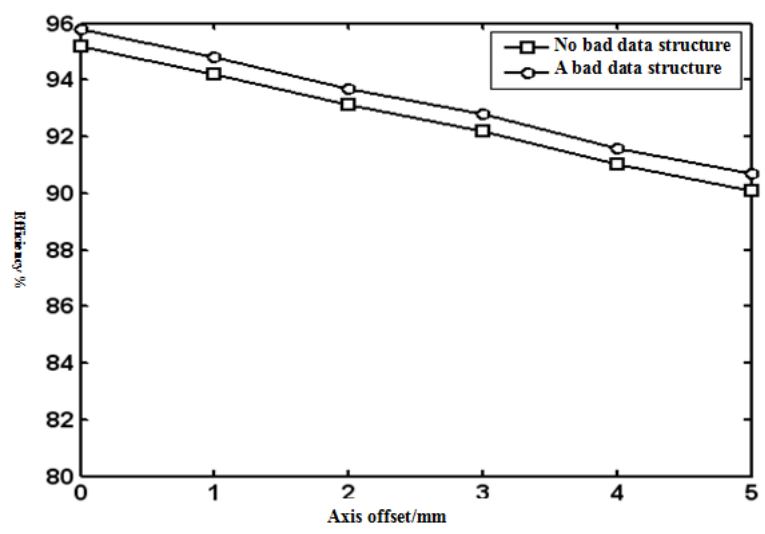

Figure 12. Experimental Data Fitting of Axis Offset and Efficiency

Use torque/speed meter to record the output torque and rotation speed of the adjustable permanent magnetic coupler in corresponding offset to calculate the corresponding transmission efficiency. Based on Figure 12, we can see that the efficiency descends with the increase of offset; small amount of axial offset creates limited influence on torque and 
efficiency, namely, when offset is at $5 \mathrm{~mm}$, the efficiency of the adjustable permanent magnetic coupler can still maintain 90\%; in condition of no displacement, when air gap is at $3 \mathrm{~mm}$, the adjustable permanent magnetic coupler can obtain its maximum power at $14 \mathrm{kw}$.

\section{Conclusion}

1) Based on the static magnetic field analysis of the adjustable permanent magnetic coupler in slotted structure, we understand that magnetic flux density is elevated in magnetic circuit and the magnetic leakage in the magnetic circuit descends. It establishes the 3D moving eddy current field to conduct dynamic analysis and we know that eddy current mainly flows along the radial direction and the its path is optimized to avoid the production of stray current;

2) Eddy current heat elevates the resistivity of the conductor disc, which leads to the re-distribution of eddy current field and reduce the transmission efficiency. In this paper, based on the experiment, it proves that the conductor disc with slotted structure is beneficial for heat radiation and it reduces the influence on eddy current field caused by elevation of resistivity of conductor disc due to eddy current heat;

3) The experiment proves that as to the two structures, under the condition of same air gap, same relative rotation speed and same axial offset, the slotted structure can elevate transmission torque; it also proves that the transmission torque in slotted structure is related to the number of ladder-shaped slots and only when the number of ladder-shaped slots is above 60, the efficiency of the slotted structure can obviously elevate the efficiency in comparison with plain structure.

\section{Reference}

[1] A. Wallace, A. Jouanne and S. Williamson, "Performance prediction and test of adjustable, permanent-magnet, load transmission systems", IEEE Industry Applications Conference 36th IAS Annual Meeting, (2001), pp. 1648-1655, Chicago.

[2] A. Wallac, A. Jouanne and A. Ramme, "A permanent-magnet coupling with rapid disconnect capability", Proceedings of International Conference on Power Electronics Machines and Drives, Bath, (2002), pp. 286-291.

[3] C. A. Smith, A. Wakeel and A. Wallace, "Formal design optimization of PM drive couplings", IEEE Industry Applications Society Annual Meeting, Pittsburgh, (2002), pp. 205-211.

[4] H. Gholizad, M. Mirsalim and M. Mirzayee, "Motional eddy currents analysis in moving solid iron using magnetic equivalent circuits method", IEEE/ACES International Conference on Wireless Communications and Applied Computational Electromagnetics, (2005), pp. 535-538, Honolulu.

[5] Y. Peng, Q. J. Ruan and Y. Zhang, "A composite grid method for moving conductor eddy current problem", IEEE Transactions on Magnetics, vol. 43, no. 7, (2007), pp. 3259-3265.

[6] A. Wallace, C. Wohlgemuth and K. Lamb, "A high efficiency, alignment and vibration tolerant, copler using high energy-product permanent magnets", Seventh International Conference on Electrical Machines and Drives, vol. 995, pp. 232-236, Durham.

[7] A. Wallace and A. Jouanne, "Industrial speed control: are PM couplings an alternative to VFDs". IEEE Industry Applications Magazine, vol. 7, no. 5, (2001), pp. 57-63.

[8] A. Wallace, A. Jouanne and R. Jeffreys, "Comparison testing of an adjustable-speed permanent-magnet eddy current coupling", Proceedings of IEEE Pulp and Paper Conference, (2000), pp. 73-78, Atlanta.

[9] X. Wang and D. Z. Wang, "Magnetic circuit structural design of Permanent Magnetic Drive", Electric Drive, vol. 41, no. 10, (2011), pp. 55-58.

[10] X. Wang, D. Z. Wang and Z. Liu, "Eddy current field analysis and performance calculations for adjustable permanent magnetic coupler", Chinese Journal of Scientific Instrument, vol. 33, no. 01, (2012), pp. 155-160.

[11] X. Wang and D. Z. Wang, "Calculation of eddy current loss and thermal analysis for adjustable permanent magnetic coupler", International Conference on Electronic and Mechanical Engineer and Information Technology, (2011).

[12] Y. Zhang, H. Zhou and Z. H. Sun, "Research of the orthoscopic permanent magnet eddy current damper in magnetic stage", International Conference on Computer Application and System Modeling, (2010).

[13] J. Alexandrova, H. Jussila, J. Nerg and J. Pyhone, "Comparison between model for eddy current loss calculations in rotor surface-mounted permanent magnets", ICEM International Conference on Electrical Machines, (2010). 
[14] B. X. Zhao and Y. C. Ji, "Analysis of flux leakage in novel permanent magnet type eddy current retarder for vehicle applications", IEEE Vehicle Power and Propulsion Conference, (2008) September 3-5.

[15] G. Cabiva, "Optimization of radial eddy current couplings", The International Journal for Computation and Mathematics in Electrical and Electronic Engineering, vol.24, no. 3, (2005), pp. 767-783.

[16] K. Hamideh, M. Razavi and U. Lamperht, "Eddy-coupling with slotted conductor disk", IEEE Transactions on magnetic, v ol. 42, no. 3, (2005) March, pp. 405-410.

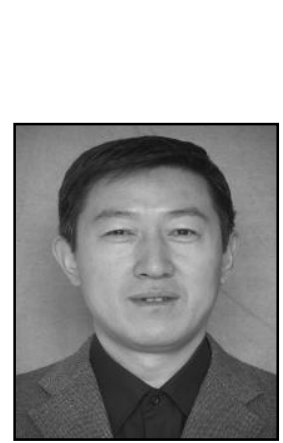

\section{Authors}

Qingzong Gao, He received his B.Sc. From Northeastern University in 2005, and received his M. Sc. from Northeastern University in 2009. Now he is a doctoral candidate in Northeastern University. His main research interest is key technologies of adjustable permanent magnetic coupler. His research interests include Speed Permanent Magnet key technologies.

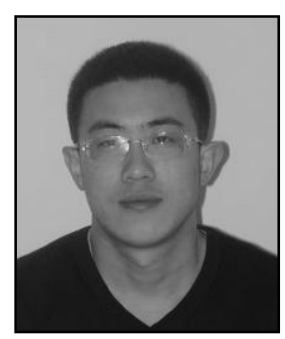

Dazhi Wang, $\mathrm{He}$ is professor and doctoral supervisor in Northeastern University, mainly engaged in the research of electric power system and electric power transmission.

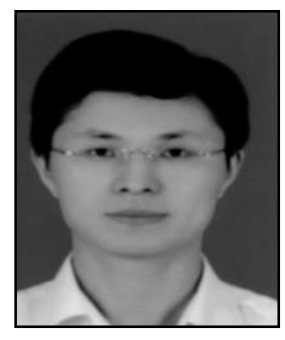

Sheng Lin, He received his B.Sc. from Northeastern University in 2005, and received his M. Sc. from Northeastern University in 2009. Now he is a doctoral candidate in Northeastern University. His main research interest is key technologies of adjustable permanent magnetic coupler. His research interests include Speed Permanent Magnet key technologies. 
International Journal of Smart Home

Vol. 9, No. 7 (2015) 